\title{
ON THE EXPERIMENTAL ANALYSIS OF INTEGRAL SLIDING MODES FOR YAW RATE AND SIDESLIP CONTROL OF AN ELECTRIC VEHICLE WITH MULTIPLE MOTORS
}

\author{
Antonio Tota ${ }^{2), 1)}$, Basilio Lenzo ${ }^{1), 3)}$, Qian $\mathrm{Lu}^{1)}$, Aldo Sorniotti1), , Patrick Gruber ${ }^{1)}$, Saber Fallah ${ }^{1)}$, \\ Mauro Velardocchia ${ }^{2}$, Enrico Galvagno ${ }^{2)}$, Jasper De Smet ${ }^{4)}$ \\ ${ }^{1}$ University of Surrey, GU2 7XH, Guildford, United Kingdom \\ ${ }^{2}$ Politecnico di Torino, Corso Duca degli Abruzzi 24, 10129, Torino, Italy \\ ${ }^{3}$ Sheffield Hallam University, S1 1WB, Sheffield, United Kingdom \\ ${ }^{4}$ Flanders MAKE, Oude Diestersebaan 133, 3920, Lommel, Belgium \\ *Corresponding author, Phone/Fax: +44 (0)1483 689688, Email: a.sorniotti@ surrey.ac.uk \\ (Received date $\quad$; Revised date $\quad$;Accepted date $\quad$
}

\begin{abstract}
With the advent of electric vehicles with multiple motors, the steady-state and transient cornering responses can be designed based on high-level reference targets, and implemented through the continuous torque control of the individual wheels, i.e., torque-vectoring or direct yaw moment control. The literature includes several papers describing the application of the sliding mode control theory to torque-vectoring. However, the experimental implementations of sliding mode controllers on real vehicle prototypes are very limited at the moment. More importantly, to the knowledge of the authors, there is lack of experimental assessments of the performance benefits of direct yaw moment control based on sliding modes, with respect to other controllers, such as the proportional integral derivative controllers or linear quadratic regulators currently used for stability control in production vehicles. This paper aims to reduce this gap by presenting an integral sliding mode controller for concurrent yaw rate and sideslip control. A new driving mode, the Enhanced Sport mode, is proposed, inducing sustained high values of sideslip angle, which can be safely limited to a specified threshold. The system is experimentally assessed on a four-wheel-drive electric vehicle along a wide range of maneuvers. The performance of the integral sliding mode controller is compared with that of a linear quadratic regulator during step steer tests. The results show that the integral sliding mode controller brings a significant enhancement of the tracking performance and yaw damping with respect to the more conventional linear quadratic regulator based on an augmented single-track vehicle model formulation.
\end{abstract}

KEY WORDS: Electric vehicle, Four-wheel-drive, Yaw rate control, Sideslip control, Integral sliding mode, Linear quadratic regulator, Experimental demonstration, Performance comparison

\section{INTRODUCTION}

Electric vehicles with individually controlled motors allow torque-vectoring (TV), i.e., continuous direct yaw moment control, to improve the cornering response in steady-state and transient conditions, and to enhance active safety. In this respect, (De Novellis et al., 2015a) compares the transient response of the same direct yaw moment controller actuated through the electric drivetrains and the friction brakes, and shows that significantly increased yaw damping is allowed by the continuous, precise and fast modulation of the electric motor torques, which is beneficial to wheel slip control as well (Savitski et al., 2016). Torque-vectoring was experimentally demonstrated in extreme transient conditions on a vehicle demonstrator with on-board electric drivetrains in (De Novellis et al., 2015b). The study defines several driving modes, selectable by the driver, each of them corresponding to a different set of understeer characteristics, thus providing a systematic approach to the specification of the TV objectives (Crolla et al., 2012).

To achieve the benefits of TV, specific control formulations are required, which can provide continuous 
and smooth control action to shape the cornering response even at low lateral accelerations. The literature includes a selection of different implementations, based on Proportional Integral Derivative (PID) controllers (De Novellis et al., 2015a; Zhang et al., 2016; Marino et al., 2010; Assadian et al., 2005), linear quadratic regulators (LQRs) (Zheng et al., 2006; Geng et al., 2009; Shino et al., 2001; Xiong et al., 2012; van Zanten, 2000), sliding mode controllers (Canale et al., 2008; Abe et al., 2001; Goggia et al., 2015a; Tchamna et al., 2013; Zhang et al., 2012; Ding et al., 2017; Thang Truong et al., 2013), $\mathrm{H}_{\infty}$ controllers (Cerone et al., 2007; Yu et al., 2013; Lu et al., 2016b), linear parameter varying controllers (Kaiser, 2014), robust controllers (Nam et al., 2014), and model predictive controllers (Falcone et al., 2007; Palmieri et al., 2012; Jalali et al., 2017), with the possibility of including fuzzy components (Geng et al., 2009) or adaptive schemes (Raksincharoensak et al., 2009). Linear or non-linear feedforward contributions can be included in the control structure (Shino et al., 2001; De Novellis et al., 2015a) to reduce the interventions of the feedback contribution, reduce sensitivity with respect to measurement errors and noise, and thus enhance drivability.

In general, TV is based on yaw rate control, with the possibility of a sideslip contribution (Manning et al., 2007). However, most of the papers including a sideslip term in their formulation do not discuss its actual benefit.
Moreover, despite the significant available literature, only a few studies (Assadian et al., 2005; De Novellis et $a l ., 2014 b$ ) compare the performance of controllers with varying levels of complexity, and unfortunately these comparisons are based on simulations. In particular, sliding mode controllers, presented in multiple recent papers, are interesting solutions for TV control, given the simplicity of their formulations, limited computational requirements and robustness. However, to the knowledge of the authors, there is a general lack of comprehensive experimental assessments of their performance, including experimental comparisons with more conventional control structures, such as the LQRs or PID controllers currently adopted for stability control systems of production vehicles (van Zanten, 2000).

This study targets this knowledge gap, with the following objectives:

- Implementation and experimental demonstration of an integral sliding mode control (ISMC) algorithm as a perturbation compensator. This is used for the concurrent control of yaw rate and sideslip angle on an electric vehicle with multiple motors. ISMC is selected for its ease of implementation and tuning, robustness with respect to matched disturbances, lack of chattering, and the fact that it represents an add-on to a more conventional and better known controller, i.e., an LQR.

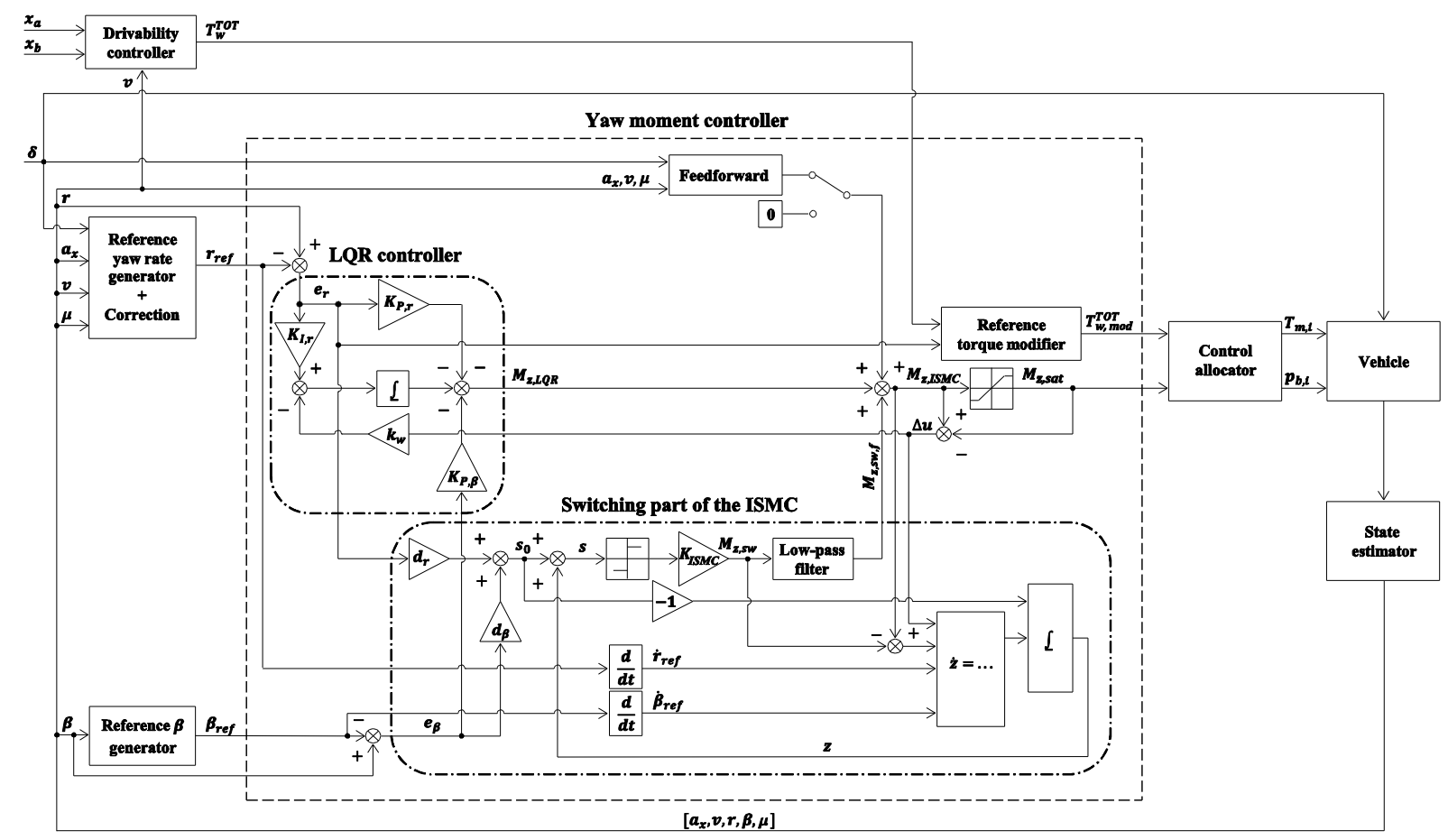

Figure 1. Simplified schematic of the vehicle control structure. 
- Experimental testing of a driving mode, i.e., the Enhanced Sport mode, purposely inducing high values of sideslip angle for increasing the 'fun-todrive', and then constraining sideslip angle at the desired threshold.

- Experimental demonstration of the performance benefit of the ISMC compared to a controller based on LQR technology with and without a non-linear feedforward contribution.

\section{CONTROL STRUCTURE AND NOMINAL LQR}

\subsection{Control structure}

Figure 1 shows the simplified schematic of the vehicle control structure, consisting of:

- A set of state estimators, e.g., providing the values of vehicle speed, $v$, sideslip angle, $\beta$, and tire-road friction coefficient, $\mu$.

- A high-level controller, generating the reference values of yaw rate and sideslip angle, respectively $r_{\text {ref }}$ and $\beta_{\text {ref }}$, based on steering wheel angle, $\delta$, vehicle speed, $v$, longitudinal vehicle acceleration, $a_{x}$, and the estimated tire-road friction coefficient, $\mu$.

- A drivability controller, generating the overall reference wheel torque, $T_{w}^{T O T}$, for traction and braking conditions, mainly based on accelerator and brake pedal positions (respectively $x_{a}$ and $x_{b}$ ), and $v$.

- A yaw moment controller, generating the reference yaw moment, $M_{z, s a t}$, to continuously track $r_{r e f}$, and constrain $\beta$ at the values specified by the high-level controller. In the case of significant yaw rate or sideslip angle errors, indicators of safety-critical conditions, this controller also modifies $T_{w}^{T O T}$ (for example, for reducing $v$ ), which becomes $T_{w, \bmod }^{T O T}$.

- A control allocation algorithm that defines the motor torque demands, $T_{m, i}$, and friction brake pressure demands, $p_{b, i}$, for the $i$-th vehicle corner (see Chen $e t$ al., 2014; Dizqah et al., 2016). In this study an equal motor torque distribution within each vehicle side is adopted for simplicity, given the focus on the ISMC performance assessment.

\subsection{Sideslip and yaw rate references}

The steady-state value of the reference yaw rate, $r_{L U T}=$ $r_{L U T}\left(\delta, v, a_{x}, \mu\right)$, is determined from a look-up table derived with a quasi-static vehicle model through the offline procedure described in (De Novellis et al., 2015a; De Novellis et al., 2015b), to achieve a reference set of understeer characteristics, i.e., the graphs of steering wheel angle as a function of lateral acceleration. The same vehicle includes multiple driving modes, such as the Normal, Sport and Enhanced Sport modes, each of them corresponding to different understeer characteristics.

$\beta_{\text {ref }}(t)$ is obtained as follows:

$\left\{\begin{array}{c}\beta_{\text {ref }}(t)=\beta(t), \text { if }|\beta(t)|<\beta_{t h}(t) \\ \beta_{\text {ref }}(t)=\beta_{t h}(t), \text { if }|\beta(t)| \geq \beta_{t h}(t)\end{array}\right.$

where $t$ is time. Based on Eq. (1), the sideslip angle contribution is always aimed at reducing $|\beta|$. In fact, when $|\beta(t)|<\beta_{t h}$, i.e., in normal driving conditions, the reference sideslip angle is coincident with the estimated sideslip angle, and only the yaw rate controller is active. In extreme maneuvering, when $|\beta(t)| \geq \beta_{t h}, \beta_{\text {ref }}(t)-$ $\beta(t)$ becomes non-zero, thus activating the sideslip controller. The threshold $\beta_{t h}(t)$ can be selected by using the phase-plane-based criteria proposed in ( $\mathrm{Lu}$ et al., 2016b). The performance of the sideslip contribution depends on the quality of the available sideslip estimation. Based on the literature and their experience (for example, see De Novellis et al., 2015a), the authors are confident that sufficiently good sideslip estimation is achievable in extreme driving conditions, which are the situations requiring the contribution of the sideslip terms of the proposed controllers.

Table 1. Simplified formulation of the reference yaw rate correction.

\begin{tabular}{ccc}
\hline \hline $\begin{array}{c}\text { Sideslip controller } \\
\text { active }\end{array}$ & $\begin{array}{c}\left|\Delta r_{\text {ref }}(t)\right| \\
\Delta \Delta r_{\text {ref,lim }}\end{array}$ & $\Delta r_{\text {ref }}(t)$ \\
\hline True & Not relevant & $k_{\text {corr }} \int \frac{M_{z, \beta}}{J_{z}} d t+\Delta r_{\text {ref,initial }}$ \\
False & True & $\int-k_{\text {ramp }} \operatorname{sign}\left(\Delta r_{\text {ref }}\right) d t+\Delta r_{\text {ref,initial }}$ \\
False & False & 0 \\
\hline \hline
\end{tabular}

In general, the sideslip-related yaw moment contribution can interfere with the yaw rate contribution, for example if the sideslip contribution is active and $\left|r_{L U T}(t)\right| \geq|r(t)|$, where $r$ is the actual yaw rate. In fact, the presence of integral control on the yaw rate error can create a windup effect when concurrent yaw rate and sideslip angle control actions are requested. This implies the need for a correction of the reference yaw rate, $\Delta r_{\text {ref }}(t)$, to support sideslip angle control, according to the criteria in Table 1. In particular, when the sideslip controller is active, $\left|r_{L U T}(t)\right|$ is varied proportionally to the integral of the sideslip-related yaw moment contribution, $M_{z, \beta}$, divided by $J_{z}$, which is the yaw mass moment of inertia of the vehicle. In fact, $M_{z, \beta} / J_{z}$ corresponds to the yaw acceleration caused by the sideslip controller, and its integral is the respective yaw 
rate variation. The initial conditions are set not to provoke discontinuities in the reference yaw rate. Thereafter, when the sideslip contribution is de-activated, the correction $\Delta r_{\text {ref }}$ is ramped down to zero at a rate defined by the parameter $k_{\text {ramp }}$ (Table 1). (Lenzo et al., 2017) discusses the details of an alternative method for modifying the reference yaw rate as a function of sideslip angle.

$r_{r e f}$ is calculated as:

$r_{r e f}=\left(r_{L U T}\left(\delta, v, a_{x}, \mu\right)+\Delta r_{r e f}\right) \frac{\omega_{r}}{p+\omega_{r}}$

The first order filter with corner frequency $\omega_{r}(p$ is the Laplace operator) is used to tune the transient response for the different driving modes.

\subsection{Nominal controller: LQR design}

The LQR of this section will be used in the remainder: i) on its own, as term of comparison of the ISMC, since LQRs are adopted in stability control systems of production vehicles (van Zanten et al., 1995; van Zanten, $2000)$; ii) in association with a non-linear feedforward contribution, generated through the procedure described in (De Novellis et al., 2015a; De Novellis et al., 2014a), and indicated as LQR+FF; and iii) as nominal controller, to which the sliding mode contribution of the ISMC as a perturbation compensator is summed.

The LQR design is based the well-known linearized single-track vehicle model (Milliken et al., 1994). The problem is formulated into a multivariable control framework, with one input (i.e., the yaw moment caused by the LQR) and two outputs (i.e., $r$ and $\beta$ ) of the plant. Kalman's controllability condition is satisfied, i.e., the determinant of the controllability matrix is different from zero, allowing the application of LQR control (Ostertag, 2011). The model is represented in augmented statespace form:

$\left\{\begin{array}{c}\dot{\boldsymbol{x}}_{a}=\boldsymbol{A}_{a} \boldsymbol{x}_{a}+\boldsymbol{B}_{a} M_{z, L Q R}+\boldsymbol{U}_{\boldsymbol{d}, \boldsymbol{a}} \\ \boldsymbol{e}=\boldsymbol{C}_{a} \boldsymbol{x}_{a}\end{array}\right.$

where $\boldsymbol{x}_{a}=\left[\begin{array}{ll}\boldsymbol{e} & \eta\end{array}\right]^{T}$ is the augmented state vector, $\boldsymbol{e}=\boldsymbol{x}-\boldsymbol{x}_{\boldsymbol{r e f}}=\left[\begin{array}{ll}\beta-\beta_{\text {ref }} & r-r_{\text {ref }}\end{array}\right]^{T}=\left[\begin{array}{ll}e_{\beta} & e_{r}\end{array}\right]^{T}$ is the error vector, $\eta$ is augmented state such that $\dot{\eta}=e_{r}$ (thus achieving an integral effect on yaw rate), $M_{z, L Q R}$ is the reference yaw moment contribution of the LQR, and $\boldsymbol{A}_{a}, \boldsymbol{B}_{a}, \boldsymbol{U}_{\boldsymbol{d}, \boldsymbol{a}}$ and $\boldsymbol{C}_{a}$ are the state-space matrices of the augmented system, which are reported in the Appendix.

The performance index, $J$, of the LQR control system design is:

$J=\frac{1}{2} \int_{0}^{\infty}\left[\boldsymbol{x}_{\boldsymbol{a}}{ }^{T} \boldsymbol{Q} \boldsymbol{x}_{\boldsymbol{a}}+R M_{z, L Q R}{ }^{2}\right] d t$

with $\boldsymbol{Q}$ and $R$ being the weighting factors related to the control tracking performance and control effort, respectively. The feedback control gain, $\boldsymbol{L}$, is obtained from:

$$
\begin{aligned}
\boldsymbol{L}=R^{-1} \boldsymbol{B}_{a}{ }^{T} \boldsymbol{P}= & {\left[K_{P, \beta} K_{P, r} K_{I, r}\right] } \\
= & {\left[\frac{p_{21}}{R J_{z}} \frac{p_{22}}{R J_{z}} \frac{p_{23}}{R J_{z}}\right] }
\end{aligned}
$$

where $\boldsymbol{P}$ is the unique positive semi-definite solution of an algebraic Riccati equation, and $p_{i j}$ is the element in the $i$-th row and $j$-th column of $\boldsymbol{P}$. Hence, $M_{z, L Q R}$ is:

$$
\begin{aligned}
M_{z, L Q R}=- & K_{P, \beta} e_{\beta}-K_{P, r} e_{r}- \\
& \int\left(K_{I, r} e_{r}-k_{w} \Delta u\right) d t
\end{aligned}
$$

$k_{w}$ is the anti-windup gain, which is multiplied by the difference, $\Delta u$, between the demanded yaw moment and the saturated yaw moment, $M_{z, s a t}$, according to the antiwindup approach in (Bohn et al., 1995; Li et al., 2011).

Based on the single-track model, the vehicle yaw dynamics are described by second order transfer functions. Their damping ratio significantly decreases with $v$ (Milliken et al., 1994). This justifies a gain scheduling design of the LQR with $v$, which is common practice in stability control systems for passenger cars. To ensure the stability of the gain scheduled controller, stability preserving interpolation is applied to the gain scheduling design. This approach can be used for arbitrary linear time invariant (LTI) controllers, providing a sufficient condition on their placement on the scheduling space, such that a stability preserving interpolated controller always exists. The formulation is in the theorem in (Stilwell et al., 1999). A set of LTI controllers $\boldsymbol{L}_{\boldsymbol{i}}(i=1, \ldots, n)$ needs to be firstly designed based on fixed values of $v$. The parameter values are selected to meet the stability covering condition. In this study six vehicle speeds (i.e., $40 \mathrm{~km} / \mathrm{h}, 60 \mathrm{~km} / \mathrm{h}, 80 \mathrm{~km} / \mathrm{h}$, $100 \mathrm{~km} / \mathrm{h}, 120 \mathrm{~km} / \mathrm{h}$ and $140 \mathrm{~km} / \mathrm{h}$ ) are used for designing the LTI controllers $\boldsymbol{L}_{i}, i=1, \ldots, 6$, which guarantee stability for the speed range $[0170 \mathrm{~km} / \mathrm{h}]$. The interpolations among the $\boldsymbol{L}_{i}$ controllers are implemented according to (Stilwell et al., 1999) for the interval [40 $\mathrm{km} / \mathrm{h} 140 \mathrm{~km} / \mathrm{h}$ ]. Below $40 \mathrm{~km} / \mathrm{h}$, the constant LTI controller $\boldsymbol{L}_{1}$ is used, while above $140 \mathrm{~km} / \mathrm{h}$ the constant LTI controller $\boldsymbol{L}_{6}$ is adopted.

The stability of the proposed LQR with respect to the variation of other parameters was verified through vehicle simulations and experimental tests, according to the industrial practice in stability control system development. The method proposed in (Lu et al., 2016a) was adopted to verify stability for significant variations of axle cornering stiffness. 


\section{ISMC DESIGN}

\subsection{ISMC as a perturbation compensator}

The ISMC formulation of this study is in terms of perturbation compensation, according to the approach discussed by (Utkin et al., 1996; Utkin et al., 1999). To use Utkin's words, in this special ISMC case "the equivalent control is generated, guaranteeing chattering alleviation and maintaining the robustness properties typical of classical sliding mode," and the "discontinuity appears only in the internal process, thus no chatters are excited in the real control path. Another advantage of this perturbation compensation scheme over the traditional methods is that the time derivative of the state vector is not necessary; the only information needed here is the upper bound of the perturbation." From the concept point of view Integral Sliding Mode is utilized here only for the estimation of the system perturbation rather than for the purpose of control. The control action to the real controlled system will be continuously enhanced by the perturbation compensator."

The ISMC implementation of this study is based on the controller in (Goggia et al., 2015a), which is extended to provide robust yaw rate control with respect to matched disturbances, and sideslip angle control when required. The gain scheduled LQR controller of Section 2.3 is used as nominal controller.

For designing the disturbance compensation part of the ISMC, a non-linear model has been selected to estimate the upper bound of the system perturbation:

$\left\{\begin{array}{c}\dot{\beta}=-r-\beta \frac{\dot{v}}{v}+\frac{F_{y}}{m v}+\frac{F_{y, d}}{m v} \\ \dot{r}=\frac{M_{z, F y}+M_{z, a l}}{J_{z}}+\frac{M_{z, F_{x}}}{J_{z}}+\frac{M_{z, d}}{J_{z}}\end{array}\right.$

where $M_{z, F_{x}}$ is the yaw moment contribution associated with the longitudinal tire forces; $M_{z, a l}$ is the yaw moment contribution associated with the aligning moments of the tires; $F_{y, d}$ and $M_{z, d}$ are the lateral force and yaw moment disturbances; and $m$ is the vehicle mass. The model in Eq (7) accounts for the variation of tire cornering stiffness as a function of the operating condition of the vehicle, which is the main limitation of the model used for LQR design. In a first approximation, the lateral tire force contribution in the vehicle reference system, $F_{y}$, is:

$F_{y}=\left(F_{x, 1}+F_{x, 2}\right) \sin \left(\delta_{w}\right)+\left(F_{y, 1}+\right.$

$\left.F_{y, 2}\right) \cos \left(\delta_{w}\right)+F_{y, 3}+F_{y, 4}$

$F_{x, i}$ and $F_{y, i}$ are the longitudinal and lateral tire forces, respectively, in the tire reference system. In particular, the subscripts ' 1 ', ' 2 ', ' 3 ' and ' 4 ' refer to the front left, front right, rear left and rear right wheels; $\delta_{w}$ is the steering angle at the wheel. The yaw moment contribution caused by the lateral tire forces, $M_{z, F y}$, is:

$$
\begin{aligned}
& M_{z, F y}=\left(F_{y, 1}+F_{y, 2}\right) a \cos \left(\delta_{w}\right)- \\
& \left(F_{y, 3}+F_{y, 4}\right) b+\left(F_{y, 1} \frac{T_{F}}{2}-F_{y, 2} \frac{T_{F}}{2}\right) \sin \left(\delta_{w}\right)
\end{aligned}
$$

where $T_{F}$ is the front track width.

The system can be re-written in the following error form:

$\dot{\boldsymbol{e}}=\boldsymbol{g}(t)+\boldsymbol{B} M_{z, s a t}(t, \boldsymbol{e})+\boldsymbol{h}(t, \boldsymbol{e})$

where $\boldsymbol{g}$ is the known part of the system; $\boldsymbol{B}=\left[\begin{array}{ll}0 & 1 /\end{array}\right.$ $\left.J_{z}\right]^{T} ; M_{z \text { sat }}$ is the saturated value of the control yaw moment $M_{z, I S M C}=M_{z, F_{x}}$; and $\boldsymbol{h}$ is the unknown part, i.e., the system perturbation. For simplicity and generality, it is assumed that no state estimator is present, and therefore it is:

$\boldsymbol{g}(t)=\left[\begin{array}{l}-\dot{\beta}_{r e f}(t) \\ -\dot{r}_{r e f}(t)\end{array}\right]$

Hence, $\boldsymbol{h}$ is defined as:

$\boldsymbol{h}=\left[\begin{array}{l}h_{\beta} \\ h_{r}\end{array}\right]=\left[\begin{array}{c}-r-\beta \frac{\dot{v}}{v}+\frac{F_{y}}{m v}+\frac{F_{y, d}}{m v} \\ M_{z, F y}+M_{z, a l}+M_{z, d} \\ J_{z}\end{array}\right]$

which includes the lateral force and yaw moment contributions due to the lateral tire forces and aligning moments. Eqs. (10)-(12) imply a conservative selection of the gains of the switching part of the ISMC. In fact, if the controller designed for the case of absence of tire force and aligning moment state estimators is effective, the same controller will be effective also for the case of state estimation (Goggia et al., 2015b).

$M_{Z, I S M C}$ consists of the sum of the nominal contribution, $M_{z, L Q R}$, related to the LQR, and the ISMC perturbation compensator term, $M_{z, s w, f}$ :

$M_{z, I S M C}=M_{z, L Q R}+M_{z, S w, f}$

$M_{z, s w, f}$ is the filtered value of a discontinuous term, $M_{Z, S w}$, calculated as a function of the sliding variable $s$ :

$s=s_{0}+z$

where $s_{0}$ is the conventional part of the sliding variable, corresponding to a linear combination (with weighting factors $d_{r}$ and $d_{\beta}$ ) of the yaw rate and sideslip angle errors:

$s_{0}=d_{r} e_{r}+d_{\beta} e_{\beta}$

The $e_{\beta}$ contribution, defined in Section 2.3, starts from an initial value equal to zero any time the sideslip contribution switches on.

$z$ is calculated through the integration of $\dot{z}$ defined as:

$\dot{z}=-\frac{\partial s_{0}}{\partial \boldsymbol{e}}\left[\boldsymbol{g}+\boldsymbol{B}\left(M_{z, I S M C}-M_{z, S w}-\Delta u\right)\right]$ 


$$
\begin{aligned}
& =\left[-d_{\beta}-d_{r}\right]\left[\begin{array}{c}
-\dot{\beta}_{r e f} \\
-\dot{r}_{r e f}+\frac{M_{z, I S M C}-M_{z, s w}-\Delta u}{J_{z}}
\end{array}\right] \\
& =d_{\beta} \dot{\beta}_{r e f}+d_{r} \dot{r}_{r e f}-d_{r} \frac{M_{z, I S M C}-M_{z, s w}-\Delta u}{J_{z}}
\end{aligned}
$$

with $z(0)=-s_{0}(\boldsymbol{e}(0))$ and $\Delta u=M_{z, I S M C}-M_{z, s a t}$. (Utkin et al., 1996) demonstrates that Eq. (16), together with the initial condition, permits to achieve sliding motion since the initial instant, without a reaching phase. Through the term $\Delta u$, this ISMC formulation includes an anti-windup effect according to the approach in (Yokoyama et al., 2010). $M_{z, s w, f}$ is given by:

$$
\begin{aligned}
M_{z, S w, f}=M_{z, S w} & \frac{\omega_{F}}{p+\omega_{F}} \\
& =-K_{I S M C} \operatorname{sign}(s) \frac{\omega_{F}}{p+\omega_{F}}
\end{aligned}
$$

where the control action is not discontinuous because of the filter with corner frequency $\omega_{F}$.

\subsection{ISMC stability}

$K_{I S M C}$ must be selected to provide stability to the system operating in uncertain conditions. To this purpose, the Lyapunov function $V_{I S M C}=\frac{1}{2} s^{2}$ is chosen.

The time derivative of $s$ can be calculated as:

$$
\begin{aligned}
\dot{s}=\dot{s}_{0}+\dot{z}=\frac{\partial s_{0}}{\partial \boldsymbol{e}} & {\left[\boldsymbol{g}+\boldsymbol{B} M_{z, s a t}+\boldsymbol{h}\right] } \\
& -\frac{\partial s_{0}}{\partial \boldsymbol{e}}[\boldsymbol{g} \\
& \left.+\boldsymbol{B}\left(M_{z, I S M C}-M_{z, s w}-\Delta u\right)\right] \\
& =\frac{\partial s_{0}}{\partial \boldsymbol{e}} \boldsymbol{h}+\frac{\partial s_{0}}{\partial \boldsymbol{e}} \boldsymbol{B} M_{z, s w}
\end{aligned}
$$

By calculating the partial derivatives of $s_{0}$ and using the definition of $M_{z, s w}$, it is:

$$
\dot{s}=d_{\beta} h_{\beta}+d_{r} h_{r}-\frac{d_{r}}{J_{z}} K_{I S M C} \operatorname{sign}(s)
$$

It follows that:

$$
\dot{V}_{I S M C}=s \dot{s} \leq|s|\left(\left|d_{\beta} h_{\beta}+d_{r} h_{r}\right|-\frac{d_{r}}{J_{z}} K_{I S M C}\right)
$$

If the uncertain terms are constrained, without any condition on their time derivative or their continuity, i.e., if $h^{*}=\left|d_{\beta} h_{\beta}+d_{r} h_{r}\right|<N$, with $N>0$, then in order to have $\dot{V}_{I S M C}<0$ for $s \neq 0$ it must be:

$K_{I S M C}>N \frac{J_{z}}{d_{r}}$

As in the practical implementation of the controller high values of $K_{I S M C}$ can bring an uncomfortable vehicle behavior in non-critical conditions, a scheduling of $K_{I S M C}$ is carried out as a function of $\left|e_{r}\right|$ and $\left|e_{\beta}\right|$; i.e., $K_{I S M C}=K_{I S M C}\left(\left|e_{r}\right|,\left|e_{\beta}\right|\right)$. In conditions of low $\left|e_{r}\right|$ and $\left|e_{\beta}\right|=0$, the value of the uncertain terms can be assumed to be low; therefore a low value of $K_{I S M C}$ is sufficient to provide system stability. The value of $N$ is obtained from the outputs of an experimentally validated vehicle model in CarMaker, used to calculate the terms of Eq. (10). Based on this analysis, the maximum value of $K_{I S M C}$ for the worst case scenario is set to $10 \mathrm{kNm} / \mathrm{rad}$. Note that:

- The non-linear model formulation of Eq. (10) is solely used to design the value of $K_{I S M C}$ through the off-line definition of the upper bound of the possible perturbation, but is not included in the on-line implementation of the controller. Hence, the nonlinear model for ISMC design does not imply any computational load for the control system hardware installed on the vehicle.

- Condition (20) ensures stability of the ISMC as a whole, including its LQR contribution, independently from the conditions in Section 2.3, such as the stability preserving interpolation, which refer to the LQR implemented on its own. ISMC stability is provided for the specified range of system perturbations, such that the larger is the possible perturbation, the larger should be $K_{I S M C}$. As a consequence, condition (20) makes the ISMC robustly stable.

\subsection{Equivalent control}

In addition to $s=0$, the condition $\dot{s}=0$ may be used to characterize the state trajectories during sliding mode. The discontinuous control action $M_{z, s w}$ in Eq. (18) represents an obstacle for the analytical calculation of the state trajectory during sliding mode. As a consequence, by disregarding the switching control action, the equivalent input, $u_{1, e q}$, can be calculated by imposing:

$\dot{s}=\frac{\partial s_{0}}{\partial \boldsymbol{e}} \boldsymbol{h}+\frac{\partial s_{0}}{\partial \boldsymbol{e}} \boldsymbol{B} u_{1, e q}=0$

which brings:

$u_{1, e q}=-\left(\frac{\partial s_{0}}{\partial \boldsymbol{e}} \boldsymbol{B}\right)^{-1} \frac{\partial s_{0}}{\partial \boldsymbol{e}} \boldsymbol{h}$

By definition, the equivalent value, $u_{1, e q}$, of a discontinuous control action is equal to the value, $M_{z, s w, f}$ in this case, output by a first order linear filter, with the discontinuous control action as input (Utkin et al., 1999). If the matrix $\frac{\partial s_{0}}{\partial \boldsymbol{e}} \boldsymbol{B}$ is non-singular during the entire system response, setting $\dot{s}=0$ reveals that $\boldsymbol{B} M_{z, s w, f}=$ $\boldsymbol{B} u_{1, e q}=-\boldsymbol{h}$ holds as well, implying that $\boldsymbol{B} M_{z, s w, f}$ is indeed an estimate of the perturbation term.

Eq. (23) brings the following system dynamics on the sliding surface:

$\dot{\boldsymbol{e}}=\boldsymbol{g}+\boldsymbol{B} M_{z, L Q R}+\boldsymbol{h}^{\prime}$

with $\boldsymbol{h}^{\prime}=\left[\begin{array}{ll}h_{\beta} & -h_{\beta} \frac{d_{\beta}}{d_{r}}\end{array}\right]^{T}$. This means that during the 
sliding motion, $h_{r}$, i.e., the so-called matched disturbance, will be rejected by the ISMC. In the case of concurrent yaw rate and sideslip control, through a specific tuning of $d_{\beta} / d_{r}$ the effect of $h_{\beta}$ (unmatched disturbance) on yaw rate control can be tuned. Methods for the compensation of unmatched disturbances through sliding mode control are proposed in (Shtessel et al., 1999). Their application to this problem will be the topic of future investigations.

\section{EXPERIMENTAL ISMC ASSESSMENT}

The ISMC as a perturbation estimator was implemented on the dSPACE AutoBox system installed on the Range Rover Evoque electric vehicle demonstrator (Figure 2) of the European Union-funded projects E-VECTOORC and iCOMPOSE. The vehicle has four on-board electric drivetrains, each consisting of a switched reluctance motor, which is connected to the wheels through a singlespeed transmission system, constant velocity joints and a half-shaft. The main vehicle parameters are reported in Table 2.

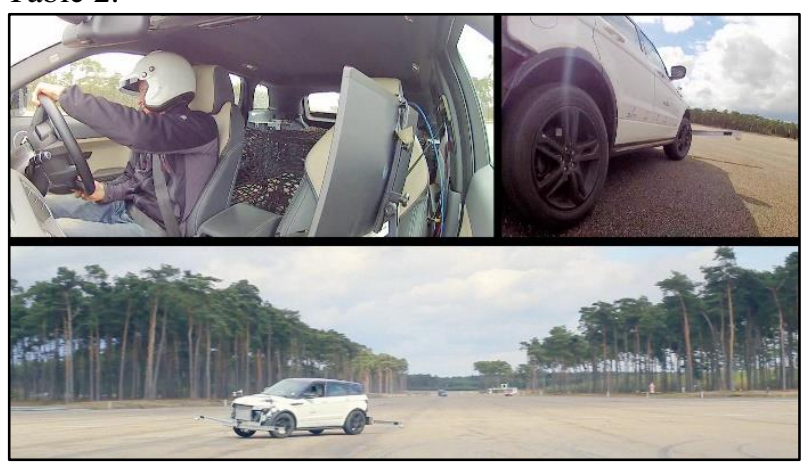

Figure 2. The four-wheel-drive electric vehicle demonstrator during a step steer test at the Lommel proving ground.

Table 2. Main vehicle parameters.

\begin{tabular}{ccc}
\hline \hline Symbol & Name and unit & Value \\
\hline$m$ & Mass (kg) & 2290 \\
$a$ & Front semi-wheelbase (m) & 1.399 \\
$l$ & Wheelbase (m) & 2.665 \\
$\tau_{g b}$ & Gearbox ratio (-) & 10.56 \\
$R_{w}$ & Wheel radius (m) & 0.364 \\
$T$ & Track width (m) & 1.616 \\
- & No. of motors per axle (-) & 2 \\
$V_{d c}$ & High-voltage dc bus level (V) & 600 \\
\hline \hline
\end{tabular}

In addition to the sensors (e.g., the steering wheel angle sensors) already present on the production vehicle used as a basis for the implementation of the demonstrator vehicle, the following sensors were installed during the session:

- The inertial measurement unit (IMU) IG-500A by SBG Systems, which includes accelerometers, gyroscopes and temperature sensors, and was used for measuring the linear vehicle accelerations and angular speeds.

- The Corrsys Datron S-350 sensor, i.e., a non-contact 2-axis optical sensor for the measurement of vehicle speed and sideslip angle.

Four maneuvers, i.e, skid pads, step steers, sequence of step steers and obstacle avoidance tests, were executed at the Lommel proving ground (Belgium) to assess the ISMC performance. The tests were performed on dry tarmac (friction coefficient of $\sim 0.9-0.95$ ) with approximantely zero road gradient and bank angle.

\subsection{Skid pad}

This test aims to show the ISMC capability of shaping the steady-state cornering response. In the skid pad test the driver slowly accelerates the vehicle and adjusts the steering wheel angle to keep the vehicle on a circular trajectory with a $60 \mathrm{~m}$ radius. The test is continued until the vehicle reaches its cornering limit.

Figure 3 compares the understeer characteristics for the passive vehicle, i.e., the vehicle with equal torque on the four wheels, and the vehicle with the ISMC in the Sport mode. The passive vehicle shows a typical non-linear cornering behavior, with an increase of the understeer gradient (i.e., the slope of the diagram) starting from lateral acceleration values of $\sim 4 \mathrm{~m} / \mathrm{s}^{2}$. On the contrary, the vehicle with the ISMC is in a condition of neutral steering throughout the whole test, consistently with the reference understeer characteristic for the selected driving mode.

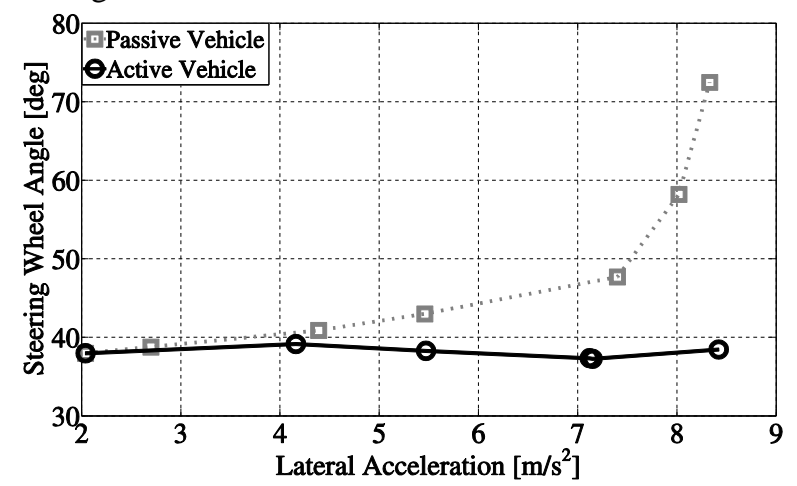

Figure 3. Examples of experimental understeer characteristics for the passive and active (Sport mode) vehicle configurations.

\subsection{Step steer}

\subsubsection{Maneuver description}

The step steer test is started from a constant speed of 100 $\mathrm{km} / \mathrm{h}$. The wheel torque demand is fixed $\left(T_{w}^{T O T}=700 \mathrm{Nm}\right.$ in this study) through the dSPACE system to avoid the 
variability of the results associated with the driver input on the accelerator pedal during the maneuver. Then the driver turns the steering wheel at a rate of $\sim 400 \mathrm{deg} / \mathrm{s}$, up to a final value of $\delta=100 \mathrm{deg}$, which is kept during the rest of the maneuver (for at least $7 \mathrm{~s}$ ). The manual steering action must ensure a high repeatability level since it directly affects the lateral dynamics. Each test was repeated at least three times and the steering angle profile was checked to ensure the correct steering wheel rate and final steering wheel angle values. A steering robot will be considered for future testing sessions (Pytka et al., 2014; AB Dynamics, 2018).

Following the steering wheel input, the increase of tire slip power losses yields a reduction of $v$ and an increase of $r$, since the vehicle is continuously operating at its maximum lateral acceleration.

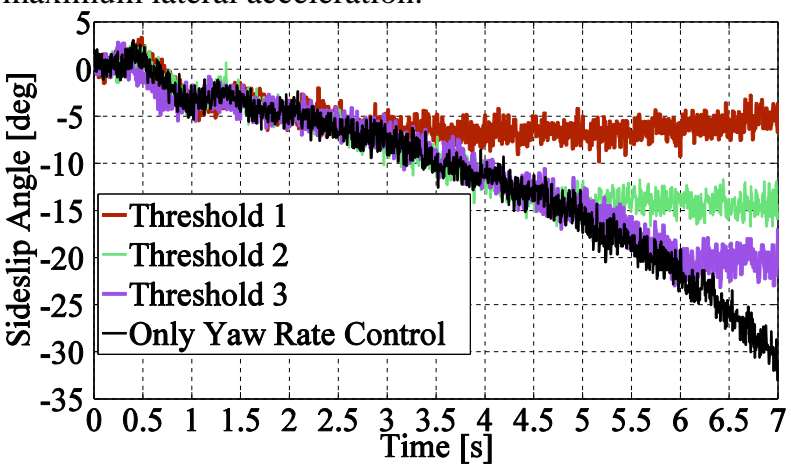

Figure 4. $\beta(t)$ during step steers with the ISMC in Enhanced Sport mode, with and without the sideslip angle controller (for different sideslip thresholds, i.e., $-7 \mathrm{deg},-14 \mathrm{deg}$, and $-21 \mathrm{deg}$ ).

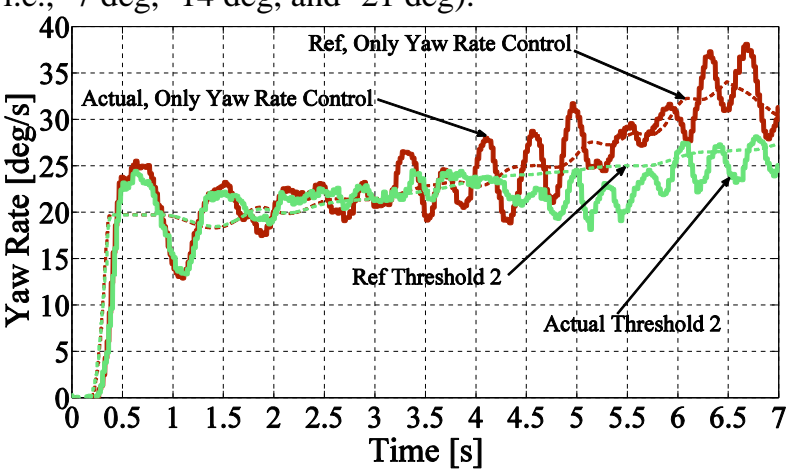

Figure 5. $r(t)$ during step steers with the ISMC in Enhanced Sport mode, with and without the sideslip angle controller.

\subsubsection{Enhanced Sport mode}

This section assesses the performance of the ISMC sideslip contribution. Figure 4 shows the results for a step steer executed in the Enhanced Sport mode with the ISMC controlling only the yaw rate, or both yaw rate and sideslip angle. The high value of yaw rate reference at the completion of the steering wheel input, i.e., in excess of the friction limits between the tires and the road surface, provokes a sideslip angle build-up. $\beta$ reaches values beyond -30 deg when the sole ISMC yaw rate controller is used. The sideslip contribution successfully limits $\beta$ to predefined thresholds (i.e, 'Threshold 1', ..., 'Threshold 3').

Figure 5 presents $r(t)$ and $r_{r e f}(t)$ for the ISMC yaw rate controller only, and the 'Threshold 2' case of the ISMC yaw rate and sideslip controller. The intervention of the sideslip contribution is associated with a reduced yaw rate (noticeable from $\sim 4 \mathrm{~s}$ onwards) compared to the vehicle with the yaw rate controller only. The reduction is caused by the concurrent effect of: i) the yaw moment required for the actuation of sideslip angle control; ii) the reference yaw rate reduction corresponding to Eq. (2) and Table 1; and iii) the higher value of $v$ caused by the lower tire slip angles.

These results demonstrate the potential sideslip control capability for 'fun-to-drive' enhancement or, conversely, for increasing active safety by allowing controlled sideslip operation at the cornering limit. The experiments also show that a relatively simple control structure can constrain sideslip angle, without having to use complex and computationally expensive non-linear model predictive control formulations.

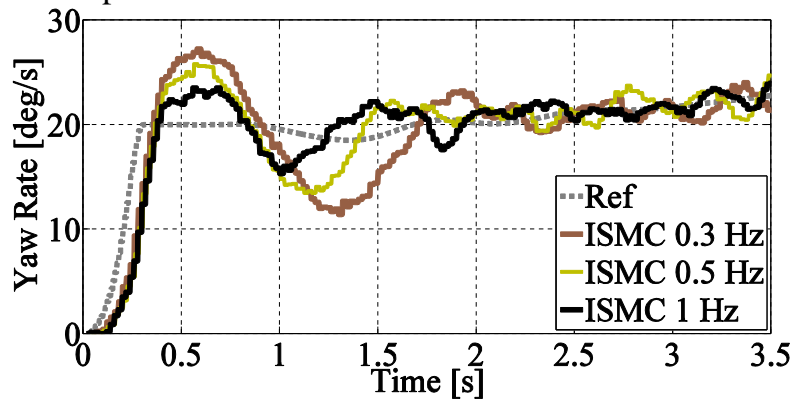

Figure 6. $r(t)$ during a step steer with the ISMC for different values of $\omega_{F}$.

\subsubsection{ISMC tunability}

For the ISMC perturbation compensator, the control system tuning is mainly based on the physically meaningful values of the gain $K_{I S M C}$ and corner frequency $\omega_{F}$. Given its limited number of parameters, the perturbation compensator contribution of the ISMC can be tuned with the conventional industrial procedures for automotive stability control systems, based on trialand-error sessions on proving grounds, after an initial setup in simulation.

For example, Figure 6 reports the variation of yaw rate response along experimental step steer tests, for different values of $\omega_{F}$. Higher values of $\omega_{F}$ increase the 'aggressiveness' of the perturbation compensator, thus originating better tracking performance, increased control effort and increased sensitivity to measurement noise. The tuning procedure of the ISMC is not more 
complex than for a typical automotive PID or LQR controller, and can be performed by a vehicle engineer without a specific know-how in sliding mode control theory.

\subsection{Sequence of step steers}

A sequence of step steers was carried out to assess the transient yaw and sideslip response in extreme conditions. The maneuver consists of: i) a first step steer at a rate of $\sim 400 \mathrm{deg} / \mathrm{s}$, to reach a value of $\delta>0$ that is kept constant for $\sim 1.5 \mathrm{~s}$; ii) a second step steer at $\sim-400 \mathrm{deg} / \mathrm{s}$, to reach a value of $\delta<0$, which has the same absolute value as that of the first step steer. This is kept constant for $\sim 1.5 \mathrm{~s}$; and iii) a final step steer that brings the system back to the final condition of $\delta=0$, at a rate of $\sim 400 \mathrm{deg} / \mathrm{s}$.

The maneuver (Figure 7) was executed for increasing values of steering wheel angle amplitude (with increments of $10 \mathrm{deg}$ ), from an initial $v$ of $100 \mathrm{~km} / \mathrm{h}$ and constant wheel torque demand imposed through the dSPACE system. The test was considered successful when $|\beta|$ remained below $10 \mathrm{deg}$ during the whole maneuver. In particular, the passive vehicle reaches this condition for a steering wheel angle amplitude of $70 \mathrm{deg}$, while the active vehicle is still within the specified sideslip boundary with an amplitude of $150 \mathrm{deg}$, which shows the significant active safety benefits of the ISMC. Since in the specific tests it was set $\beta_{t h}=15 \mathrm{deg}$, the intervention of the sideslip angle contribution was not even needed.

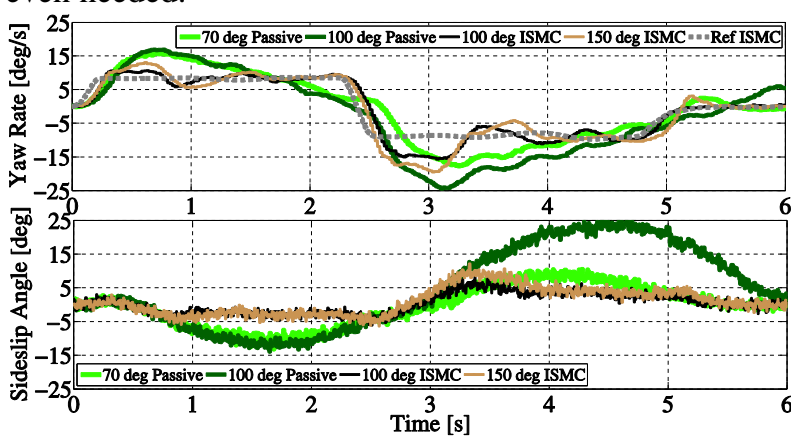

Figure 7. $r(t)$ and $\beta(t)$ for the passive and active (Normal mode) vehicles during sequences of step steers with a sideslip threshold $\beta_{t h}=15 \mathrm{deg}$.

\subsection{Obstacle avoidance test}

The obstacle avoidance test (ISO 3888-2, 2011) was carried out to subjectively investigate the road-holding ability of the vehicle. After the initial stabilization at the speed set for the specific test, a constant total wheel torque demand of $200 \mathrm{Nm}$ was imposed through the dSPACE unit. The driver had to control the steering wheel input to keep the vehicle within a trajectory set by cones, defining: i) an initial lane; ii) a second lane with a lateral offset with respect to the initial lane; and iii) a final lane approximately aligned with the initial lane.

Figure 8 shows that the controlled vehicle requires a significantly reduced steering correction after the first lane change, when the driver has to stabilize the vehicle to keep it within the second lane without hitting the cones. Correspondingly, the yaw rate and sideslip angle oscillations are reduced, and thereby the vehicle exhibits a better performance. Also, owing to the lower values of $|\beta(t)|$, the vehicle with the ISMC maintains a higher speed during the maneuver.

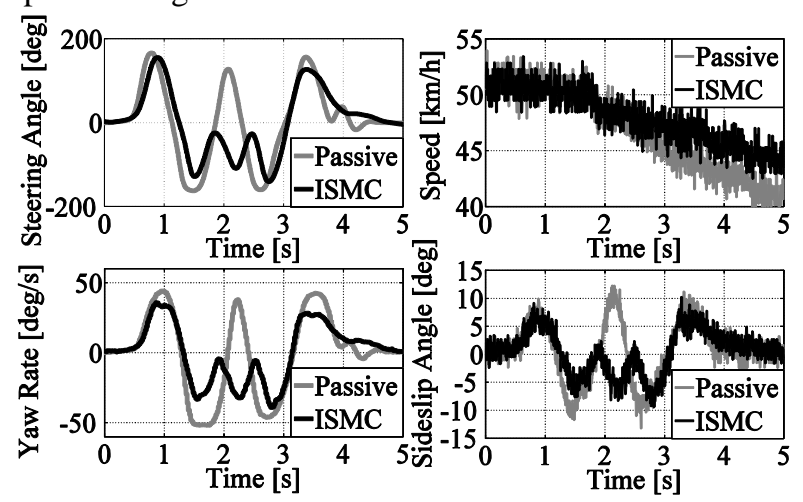

Figure 8. $\delta(t), r(t)$ and $\beta(t)$ for the passive and active (in Normal mode) vehicles during an obstacle avoidance maneuver from an initial $v=51.5 \mathrm{~km} / \mathrm{h}$.

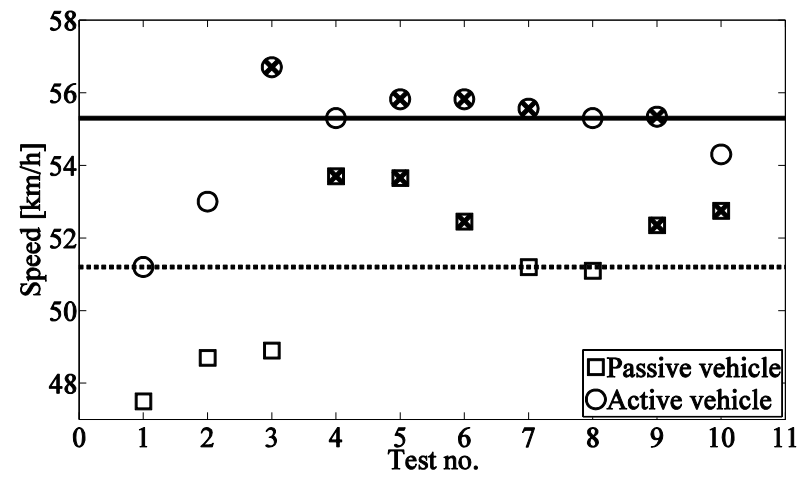

Figure 9. Distribution of the successful (indicated by the blank symbols) and unsuccessful (indicated by ' $x$ ') tests for the passive and active (in Normal mode) vehicles during obstacle avoidance maneuvers.

Figure 9 reports the results for a sample of tests. The figure indicates the corresponding initial speed, i.e, the speed at the entrance of the first lane, and whether the test was successful or unsuccessful. The test is considered successful when the vehicle performs the maneuver without hitting any cone placed along the boundaries of the obstacle avoidance track. The results show a 7\% increase of the maximum initial speed of the successful tests with the controlled vehicle with respect to the passive vehicle. The higher speed demonstrates the 
controller benefits in terms of enhanced vehicle handling qualities and active safety.

\section{EXPERIMENTAL COMPARISON OF THE ISMC, LQR AND LQR+FF}

The key objective of this study is to experimentally assess whether the ISMC brings a performance benefit with respect to more conventional control structures, based on LQR technology, currently used for stability control systems actuating the friction brakes. Such an evaluation, absent so far in the literature, is necessary to promote the industrial development of sliding mode implementations for torque-vectoring.

Figure 10 shows the time histories of vehicle yaw rate during a step steer test for: a) the passive vehicle; b) the vehicle controlled with the sole LQR; c) the vehicle with the same LQR as in b) and the non-linear static feedforward contribution ( $\mathrm{LQR}+\mathrm{FF}$ ); and d) the vehicle with the ISMC, including the same LQR as in b) as nominal controller.

In Figure 10 all controllers reduce the duration of the yaw rate oscillations following the steering wheel input. The performance of the ISMC is particularly effective in decreasing the first yaw rate overshoots and undershoots, which are $16.8 \mathrm{deg} / \mathrm{s}$ and $24.5 \mathrm{deg} / \mathrm{s}$ in a), $10.1 \mathrm{deg} / \mathrm{s}$ and $8.4 \mathrm{deg} / \mathrm{s}$ in b), 11.1 and $7.0 \mathrm{deg} / \mathrm{s}$ in c), and $3.5 \mathrm{deg} / \mathrm{s}$ and $4.1 \mathrm{deg} / \mathrm{s}$ in $\mathrm{d})$.

Table 3 includes the values of the four objective performance indicators used to assess the controllers along the maneuver, i.e:

- The yaw rate overshoot, $O S_{\%}$, calculated as:

$$
O S_{\%}=\frac{r\left(t_{\text {peak,f }}\right)-r_{\text {ref }}\left(t_{\text {peak }, f}\right)}{r_{\text {ref }}\left(t_{\text {peak }, f}\right)} 100
$$

where $t_{\text {peak,f }}$ is the time at which the first yaw rate peak is achieved after the steering input is applied. For drivers with average skills, this peak corresponds to an unexpected, and potentially dangerous, vehicle behavior. The ISMC performance enhancement with respect to the $\mathrm{LQR}$ and $\mathrm{LQR}+\mathrm{FF}$ is of the same order of magnitude of the performance enhancement of the LQR and LQR+FF with respect to the passive vehicle. This is an important conclusion of this study, which encourages the further industrial evaluation of ISMC as a perturbation compensator for TV control.

- The root mean square value of the yaw rate error, $R M S E$, calculated during the $3 \mathrm{~s}$ following the application of the steering wheel input. The ISMC is able to reduce the RMSE by $77 \%, 49 \%$, and $42 \%$, in comparison with the cases a), b), and c), respectively.

- The integral of the absolute value of the control action, $I A C A$, normalized with time, defined as:

\section{IACA}

$$
=\frac{1}{t_{\text {man,fin }}-t_{\text {man }, \text { in }}} \int_{t_{\text {man }, \text { in }}}^{t_{\text {man }, \text { fin }}}\left|M_{z, \text { sat }}(t)\right| d t
$$

and calculated during the relevant part of the maneuver, i.e., between the times $t_{\text {man,in }}$ and $t_{\text {man,fin }}$ (in this case the $3 \mathrm{~s}$ following the steering application). The significantly improved performance of the ISMC corresponds to a marginal increase of the control effort, $13 \%$ and $6 \%$ higher than for the LQR and LQR+FF controllers, respectively.

- The delay between the reference yaw rate and the actual yaw rate, $t_{d}$, evaluated for $r_{r e f}=15 \mathrm{deg} / \mathrm{s}$. This indicator is approximately $30 \%$ lower for all controlled vehicles, indicating enhanced responsiveness during transients. $t_{d}$ can be effectively designed by tuning the corner frequency, $\omega_{r}$, of the filter providing the reference yaw rate.
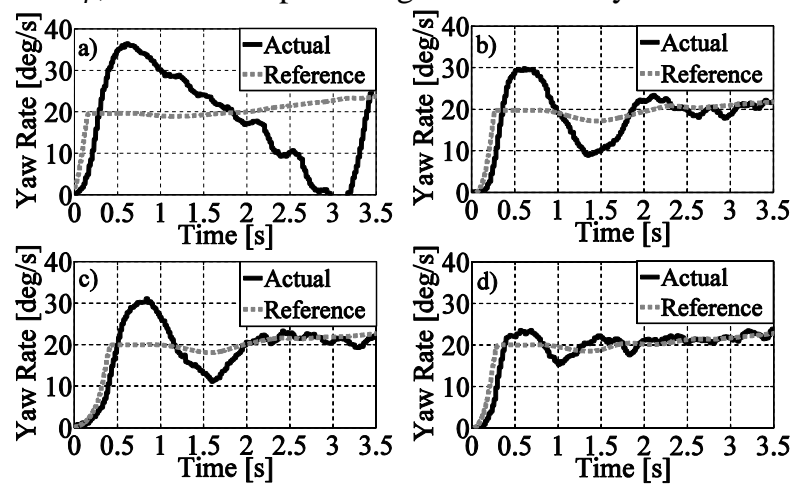

Figure 10. $r(t)$ during a step steer for the passive (a) and active (LQR (b), LQR+FF (c) and ISMC (d)) vehicles.

Table 3. Performance indicators for the step steer test for the passive and controlled vehicles.

\begin{tabular}{ccccc}
\hline \hline & $O S_{\%}$ & $R M S E$ & $I A C A$ & $t_{d}$ \\
& {$[\%]$} & {$[\mathrm{deg} / \mathrm{s}]$} & {$[\mathrm{Nm}]$} & {$[\mathrm{s}]$} \\
\hline Baseline & 85.55 & 11.45 & - & 0.12 \\
LQR & 51.49 & 5.175 & 1578 & 0.09 \\
LQR + FF & 55.76 & 4.545 & 1675 & 0.09 \\
ISMC & 17.51 & 2.634 & 1780 & 0.09 \\
\hline \hline
\end{tabular}

Figure 11 shows $M_{z, s a t}(t)$ for the three controllers. For all tests the maximum yaw moment was limited to $4000 \mathrm{Nm}$. The results indicate that the ISMC generates the first negative (stabilizing) peak of yaw moment earlier and for a longer duration than the other two controllers. In doing so, the ISMC is able to reduce the first yaw rate overshoot as mentioned above.

Figure 12 reports the time histories of the yaw moment contributions of the ISMC; in particular the proportional term of the $L Q R$ ('LQR ${ }_{P r}$ ' in the figure), the integral term of the LQR (' $L Q R_{I r}$ '), the switching contribution, ('Switching ISMC'), and their sum ('Total'). The proportional term is the main contributor for the reduction of the time delay in the initial yaw rate build- 
up phase, while the switching ISMC contribution, i.e., the perturbation compensator, is primarily responsible for the vehicle stabilization between $0.4 \mathrm{~s}$ and $0.8 \mathrm{~s}$. As during this test the sideslip angle remains consistently low, the sideslip contribution is inactive.

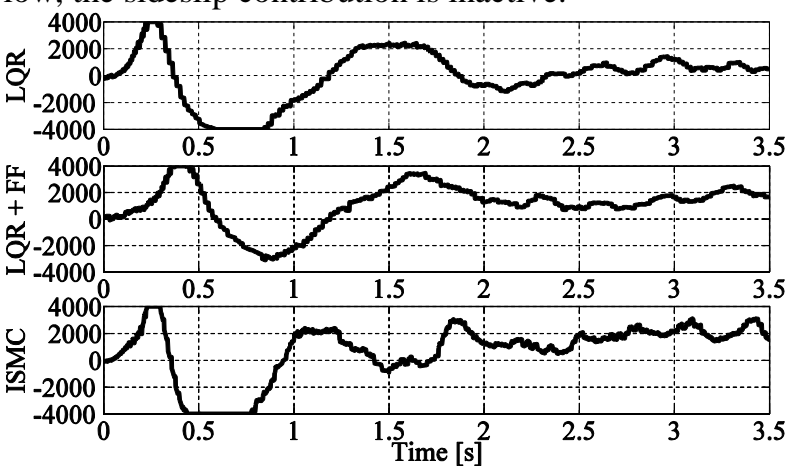

Figure 11. $M_{z, s a t}(t)$ during a step steer for the LQR, $\mathrm{LQR}+\mathrm{FF}$ and ISMC.

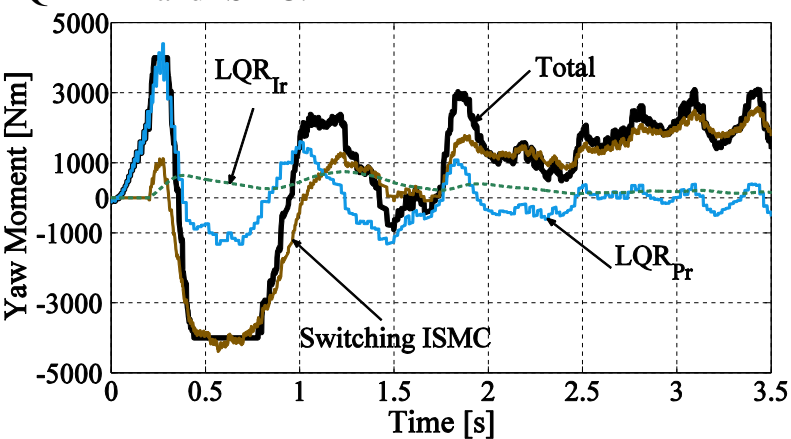

Figure 12. ISMC yaw moment contributions during a step steer.

\section{CONCLUSION}

This study discussed an integral sliding mode controller for the concurrent control of yaw rate and sideslip angle on a four-wheel-drive electric vehicle with on-board drivetrains. The sliding mode controller was implemented as a perturbation compensator added to a nominal linear quadratic regulator in order to provide robustness with respect to matched disturbances. The comprehensive set of experimental results in steady-state and transient conditions yields the following conclusions:

- The continuous actuation of yaw rate control allows very different understeer characteristics for the same vehicle, depending on the selected driving mode.

- The sideslip yaw moment contribution is useful to control vehicle response in extreme conditions, such as those induced by the Enhanced Sport mode or by an overestimation of the tire-road friction coefficient. For all these conditions, the proposed sideslip controller is effective in limiting the sideslip angle to a specified threshold.
- The switching contribution of the ISMC as a perturbation compensator significantly enhances the controller tracking performance in transient conditions compared to a linear quadratic regulator with augmented states, used as nominal controller within the ISMC. More specifically, according to the adopted performance indicators, the benefits associated with the ISMC perturbation compensator with respect to the nominal LQR controller on its own are comparable to the benefits of the LQR controllers with respect to the baseline vehicle. This is a significant novel result of this study.

- The simple formulation and tunability of the ISMC structure, without the need for a feedforward contribution, facilitates its industrial implementation on real vehicles.

ACKNOWLEDGEMENT - The research leading to these results has received funding from the European Union Seventh Framework Programme FP7/2007-2013 under grant agreement no. 608897.

\section{APPENDIX}

The augmented state-space formulation of the system is given by:

$\left\{\begin{array}{c}\dot{\boldsymbol{x}}_{a}=\boldsymbol{A}_{a} \boldsymbol{x}_{a}+\boldsymbol{B}_{a} M_{z, L Q R}+\boldsymbol{U}_{\boldsymbol{d}, \boldsymbol{a}} \\ \boldsymbol{e}=\boldsymbol{C}_{a} \boldsymbol{x}_{a}\end{array}\right.$

where the matrices $\boldsymbol{A}_{a}, \boldsymbol{B}_{a}$ and $\boldsymbol{C}_{a}$ are defined as:

$\boldsymbol{A}_{a}=\left[\begin{array}{ll}\boldsymbol{A} & \mathbf{0} \\ \boldsymbol{F} & 0\end{array}\right], \boldsymbol{B}_{a}=\left[\begin{array}{l}\boldsymbol{B} \\ 0\end{array}\right], \boldsymbol{C}_{a}=\left[\begin{array}{ll}\boldsymbol{C} & \mathbf{0} \\ \mathbf{0} & 0\end{array}\right]$

It is $\boldsymbol{F}=\left[\begin{array}{ll}0 & 1\end{array}\right]$, while $\boldsymbol{A}, \boldsymbol{B}, \boldsymbol{C}$ are the matrices of the state-space formulation of the conventional single-track model, i.e., without the augmented state:

$\boldsymbol{A}=\left[\begin{array}{cc}-\frac{1}{m v}\left(C_{f}+C_{r}\right) & -1-\frac{1}{m v^{2}}\left(a C_{f}-b C_{r}\right) \\ -\frac{1}{J_{z}}\left(a C_{f}-b C_{r}\right) & -\frac{1}{v J_{z}}\left(a^{2} C_{f}+b^{2} C_{r}\right)\end{array}\right]$,

$\boldsymbol{B}=\left[\begin{array}{l}0 \\ 1 \\ J_{z}\end{array}\right], \quad \boldsymbol{C}=\left[I_{2 \times 2}\right]$

$m$ is the vehicle mass; $a$ and $b$ are the front and rear semi-wheelbases; $C_{f}$ and $C_{r}$ are the front and rear axle cornering stiffness. $\boldsymbol{U}_{\boldsymbol{d}, \boldsymbol{a}}$ is the disturbance term of the augmented system, defined as:

$\boldsymbol{U}_{\boldsymbol{d}, \boldsymbol{a}}=\boldsymbol{A}_{r} \boldsymbol{x}_{\boldsymbol{r e f , a}}-\boldsymbol{I}_{a}\left(\dot{\boldsymbol{x}}_{\boldsymbol{r e f}, \boldsymbol{a}}+\boldsymbol{d}\right)+\boldsymbol{E}_{a} \delta_{w}$

with $\boldsymbol{x}_{\boldsymbol{r e f}, \boldsymbol{a}}=\left[\begin{array}{lll}\beta_{\text {ref }} & r_{r e f} & 0\end{array}\right]^{T} ; \delta_{w}$ is the steering angle at the wheel; and $\boldsymbol{d}$ represents model uncertainties and exogenous disturbances. $\boldsymbol{A}_{r}, \boldsymbol{I}_{\boldsymbol{a}}$ and $\boldsymbol{E}_{\boldsymbol{a}}$ are given by:

$\boldsymbol{A}_{r}=\left[\begin{array}{ll}\boldsymbol{A} & \mathbf{0} \\ \mathbf{0} & 0\end{array}\right], \boldsymbol{I}_{a}=\left[\begin{array}{cc}\boldsymbol{I}_{2 x 2} & \mathbf{0} \\ \mathbf{0} & 0\end{array}\right], \boldsymbol{E}_{a}=\left[\begin{array}{c}\frac{1}{m v} C_{f} \\ \frac{a}{J_{z}} C_{f} \\ 0\end{array}\right]$ 


\section{REFERENCES}

AB Dynamics. [online] Available at: https://www.abdynamics.com/en/products/tracktesting/driving-robots/steering-robots [Accessed on 26 March 2018]

Abe M., Kano Y., Suzuki K., Shibahata Y., Furukawa Y. (2001). Sideslip control to stabilize vehicle lateral motion by direct yaw moment, JSAE, Vol. 22(9), pp. 413 419.

Assadian F., Hancock M. (2005). A Comparison of Yaw Stability Control Strategies for the Active Differential, IEEE Symposium on Industrial Electronics.

Bohn C., Atherton D.P. (1995). An Analysis Package Comparing PID Anti-Windup Strategies, IEEE Transactions on Control Systems, Vol. 15(2), pp. 34-40.

Canale M., Fagiano L., A. Ferrara A., Vecchio C. (2008). Vehicle yaw control via second-order slidingmode technique, IEEE Transactions on Industrial Electronics, Vol. 55(11), pp. 3908-3916.

Cerone V., Milanese M., Regruto D. (2007). Yaw stability control design through a mixed sensitivity approach, IEEE Intelligent Vehicles Symposium.

Chen Y., Wang J. (2014). Design and Experimental on Energy Efficient Control Allocation Methods for Overactuated Electric Vehicles: Longitudinal Motion Case, IEEE/ASME Transactions on Mechatronics, Vol. 19(2), pp. 538-548.

Crolla D.A., Cao D. (2012). The impact of hybrid and electric powertrains on vehicle dynamics, control systems and energy regeneration, Vehicle System Dynamics, Vol. 50(1), pp. 95-109.

De Novellis L., Sorniotti A., Gruber P. (2014a). Wheel Torque Distribution Criteria for Electric Vehicles with Torque-Vectoring Differentials, IEEE Transactions on Vehicular Technology, Vol. 63(4), pp. 1593-1602.

De Novellis L., Sorniotti A., Gruber P., Pennycott A. (2014b). Comparison of Feedback Control Techniques for Torque-Vectoring Control of Fully Electric Vehicles, IEEE Transactions on Vehicular Technology, Vol. 63(8), pp. 3612-3623.

De Novellis L., Sorniotti A., Gruber P., Orus J., Rodriguez Fortun J.M., Theunissen J., De Smet J. (2015a). Direct yaw moment control actuated through electric drivetrains and friction brakes: theoretical design and experimental assessment, Mechatronics, Vol. 26, pp. $1-15$.

De Novellis L., Sorniotti A., Gruber P. (2015b). Driving modes for designing the cornering response of fully electric vehicles with multiple motors, Mechanical Systems and Signal Processing, Vol. 64-65, pp. 1-15.

Ding S., Liu L., Zheng W.X. (2017). Sliding Mode Direct Yaw-Moment Control Design for In-wheel Electric Vehicles, IEEE Transactions on Industrial Electronics, Vol. 64(8), pp. 6752-6762.
Dizqah A.M., Lenzo B., Sorniotti A., Gruber P., De Smet J. (2016). A Fast and Parametric Torque Distribution Strategy for Four-Wheel-Drive Energy Efficient Electric Vehicles, IEEE Transactions on Industrial Electronics, Vol. 63(7), pp. 4367-4376.

Falcone P., Borrelli F., Asgari J., Tseng E.H., and Hrovat D. (2007). Predictive Active Steering Control for Autonomous Vehicle Systems, IEEE Transactions on Control System Technology, Vol. 15(3), pp. 566-580.

Geng C., Mostefai L., Denaï M., Hori Y. (2009). Direct Yaw-Moment Control of an In-Wheel-Motored Electric Vehicle Based on Body Slip Angle Fuzzy Observer, IEEE Transactions on Industrial Electronics, Vol. 56(5), pp. 1411-1419.

Goggia T., Sorniotti A., De Novellis L., Ferrara A., Gruber P., Theunissen J., Steenbeke D., Knauder B., Zehetner J. (2015a). Integral Sliding Mode for the Torque-Vectoring Control of Fully Electric Vehicles: Theoretical Design and Experimental Assessment, IEEE Transactions on Vehicular Technology, Vol. 64(5), pp. 1701-1715.

Goggia T., Sorniotti A., Ferrara A., De Novellis L., Pennycott A., Gruber P., Yunus I. (2015b). Integral Sliding Mode for the Yaw Moment Control of FourWheel-Drive Fully Electric Vehicles with In-Wheel Motors, International Journal of Powertrains, Vol.4(4), pp. $388-419$.

ISO 3888-2. (2011). Passenger cars - Test track for a severe lane-change manoeuvre - Part 2: Obstacle avoidance.

Jalali M., Ashemi E., Khajepour A., Chen S., and Litkouhi B. (2017). Integrated model predictive control and velocity estimation of electric vehicles, Mechatronics, Vol. 46, pp. 84-100.

Kaiser G. (2014). Torque Vectoring - Linear Parameter-Varying Control for an Electric Vehicle, PhD Thesis, Hamburg-Harburg Technical University.

Lenzo B., Sorniotti A., Gruber P., Sannen K. (2017). On the experimental analysis of single input single output control of yaw rate and sideslip angle, International Journal of Automotive Technology, Vol. 18(5), pp. 799811.

Li X., Park J., Shin H. (2011). Comparison and Evaluation of Anti-Windup PI Controllers, Journal of Power Electronics, Vol. 11(1), pp. 45-50.

Lu Q., Sorniotti A., Gruber P., Theunissen J., De Smet J. (2016a). Hळ loop shaping for the torque-vectoring control of electric vehicles: Theoretical design and experimental assessment, Mechatronics, Vol. 35, pp. 3243.

Lu Q., Gentile P., Tota A., Sorniotti A., Gruber P., Costamagna F., De Smet J. (2016b). Enhancing vehicle cornering limit through sideslip and yaw rate control, Mechanical Systems and Signal Processing, Vol. 75, pp. 455-472. 
Manning W.J., Crolla D.A. (2007). A review of yaw rate and sideslip controllers for passenger vehicles, Transactions of the Institute of Measurement and Control, Vol. 29(2), pp. 117-135.

Marino R., Scalzi S. (2010). Asymptotic sideslip angle and yaw rate decoupling control in four-wheel steering vehicles, Vehicle System Dynamics, Vol. 48(9), pp. 9991019.

Milliken W.F., Milliken D.L., Race Car Vehicle Dynamics, SAE International, 1994.

Nam K., H. Fujimoto, Hori. Y. (2014). Advanced Motion Control of Electric Vehicles Based on Robust Lateral Tire Force Control via Active Front Steering, IEEE/ASME Transactions on Mechatronics, Vol. 19(1), pp. 289-299.

Ostertag E. (2011). Mono-and Multivariable Control and Estimation, Springer.

Palmieri G., Barić M., Glielmo L., Borrelli F. (2012). Robust vehicle lateral stabilisation via set-based methods for uncertain piecewise affine systems, Vehicle System Dynamics, Vol. 50(6), pp. 861-882.

Pytka J.A. (2014). Effects of steering dynamics upon tyre lateral forces on deformable surfaces. International Journal of Vehicle Design, Vol. 64(2/3/4), pp. 170-194.

Raksincharoensak P., Mizushima T., Nagai M. (2009). Direct yaw moment control system based on driver behavior recognition, Vehicle System Dynamics, Vol. 46(Supp. 1), pp. 911-921.

Savitski D., Ivanov V., Shyrokau B., Puetz T., De Smet J. (2016). Experimental investigations on continuous regenerative anti-lock braking system of full electric vehicle, International Journal of Automotive Technology, Vol. 17(2), pp. 327-338.

Shino M., Nagai M. (2001). Yaw-moment control of electric vehicle for improving handling and stability, JSAE, Vol. 22, pp. 473-480.

Shtessel Y., Edwards C., Fridman L., Levant A. (1999). Sliding Mode Control and Observation, Birkhauser.

Stilwell D.J., Rugh W.J. (1999). Interpolation of observer state feedback controller for gain scheduling, IEEE Transactions on Automatic Control, Vol. 44(6), pp. 1225-1229.
Tchamna R., Youn I. (2013). Yaw Rate and Side-Slip Control Considering Vehicle Longitudinal Dynamics, International Journal of Automotive Technology, Vol. 14(1), pp. 53-60.

Thang Truong D.V., Meywerk M., Tomaske W. (2013). Torque Vectoring for Rear Axle using Adaptive Sliding Mode Control, International Conference on Control, Automation and Information Sciences.

Utkin V., Shi J. (1996). Integral sliding mode in systems operating under uncertainty conditions, IEEE Conference on Decision and Control.

Utkin V., Guldner J., Shi J. (1999). Sliding Mode Control in Electromechanical Systems. New York, NY, USA: Taylor \& Francis.

Xiong L., Yu Z., Wang Y., Yang C., Meng T. (2012). Vehicle dynamics control of four in-wheel motor drive electric vehicle using gain scheduling based on tyre cornering stiffness estimation, Vehicle System Dynamics, Vol. 50(6), pp. 831-846.

Yokoyama M., Kim G., Tsuchiya M. (2010). Integral Sliding Mode Control with Anti-Windup Compensation and its Application to a Power Assist System, Journal of Vibration and Control, Vol. 16(4), pp. 503-512.

Yu H., Huang M., Zhang Z. (2013). Direct YawMoment H-infinity Control of Motor-Wheel Driving Electric Vehicle, IEEE Vehicle Power and Propulsion Conference.

van Zanten A., Erhardt R., Pfaff G. (1995). VDC: The Vehicle Dynamics Control System of Bosch, SAE Technical Paper 950759.

van Zanten A. (2000). Bosch ESP systems: 5 years of experience, SAE Technical Paper 2000-01-1633.

Zhang H., Zhang J. (2012). Yaw Torque Control of Electric Vehicle Stability, IEEE $6^{\text {th }}$ International Conference on Information and Automation for Sustainability.

Zhang H., Wang J. (2016). Vehicle Lateral Dynamics Control Through AFS/DYC and Robust GainScheduling Approach, IEEE Transactions on Vehicular Technology, Vol. 65(1), pp. 489-494, 2016.

Zheng S., Tang H., Han Z., Zhang Y. (2006). Controller design for vehicle stability enhancement, Control Engineering Practice, Vol. 14, pp. 1413-1421. 